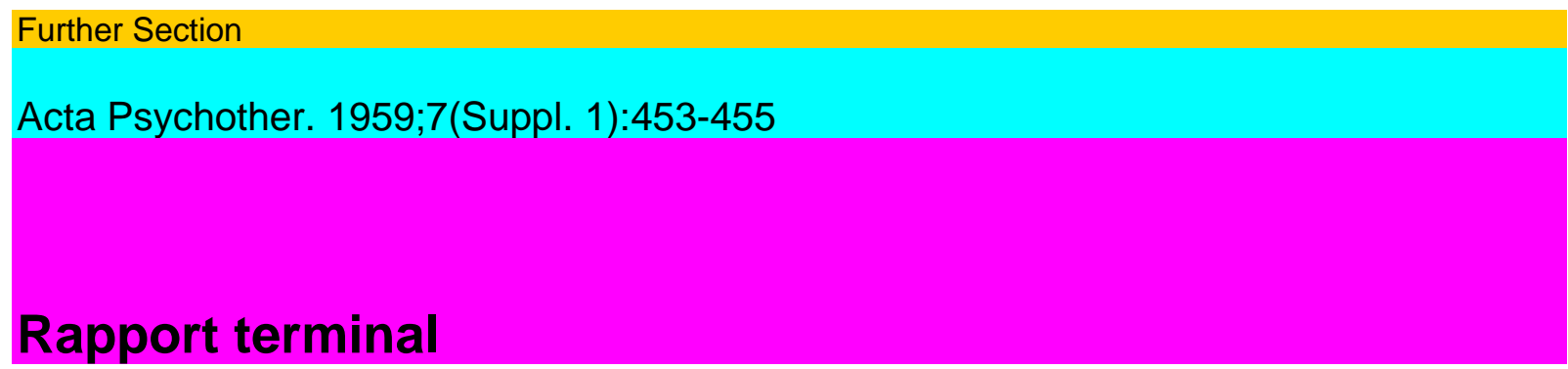

Au moment où j'ai Thonneur de prendre la parole pour tenter en quelques minutes de vous dire mes sentiments sur les travaux de votre Congrès, je dois vous avouer que je n'étais pas sans apprehension il y a quelques jours, avant le debut de nos travaux. Vous venez de vingt-huit pays et vous parlez des langues différentes. Nous avons vu tout au long de ces trois journées les difficultés qu'il y avait à communiquer entre nous. Vous appartenez à des professions nombreuses et variées: psychiatres, psychologues, travailleurs sociaux, professeurs et pedagogues, économistes et j'en passe. Enfin vos conceptions théoriques et techniques sur la psychothérapie sont différentes: beaucoup d'entre nous appartiennent à Гécole psychanalytique; d'autres se réclament des theories psychodramatiques. D'autres enfin, sans adherer à aucune conception particulière, tendent à attacher plus d'importance aux conceptions assez diverses de la psychologie dynamique. Y aurait-il un terrain d'entente entre nous, voilà la question que je me posais. Après tout la psychothérapie de groupe existait-elle de façon spécifique et n'était-ce pas qu'une variété de psychothérapie pratiquée en groupe par des praticiens se réclamant de theories et de techniques diverses?

Au moment où notre Congrès se termine, je crois pourtant que nous pouvons tous affirmer que nous avons eu des journées intéressantes. II me semble qu'au terme de ces trois jours, on peut affirmer que la psychothérapie de groupe existe bien et je vais essayer de le montrer au travers de quelques reflexions.

Tout d'abord les travaux de notre Congrès ont mis en cause, me semble-t-il, la notion même de groupe. Or il paraît à Гévidence que les congressistes ont vécu une experience très positive de vie et de discussion en groupe. Je me suis laissé dire que les travaux dans les sections et surtout dans les séminaires ont été souvent fructueux. Un fait assez remarquable est que les discussions dans les sections semblent avoir été plus animées et plus vivantes le deuxième jour, c'est-à-dire après que les congressistes aient eu Гoccasion de discuter librement, sans aucun theme préalable, dans les séminaires de travail. La vie de groupe s'est affirmée dans bien des reactions dont je ne citerai que Гune: beaucoup d'entre nous ont échangé des adresses, donnant ainsi des perspectives futures à la vie des divers petits groupes que nous avons formes et il serait souhaitable que nous puissions connaître les adresses des uns et des autres grace à Torganisation permanente du Comité international de psychothérapie de groupe dont cela me paraît pouvoir constituer une tâche intéressante.

Dans nos travaux nous avons mis également en cause la notion de psychothérapie de groupe. Sans aucun doute des dynamiques qui soustendent les différentes techniques que nous pouvons appliquer paraissent assez différentes. De son côté, Moreno en valorisant la notion de télé, en étudiant les forces de cohesion du groupe, en racontant d'une manière particulièrement illustrative, les moyens d'expression dramatique que celui-ci peut employer, a montré

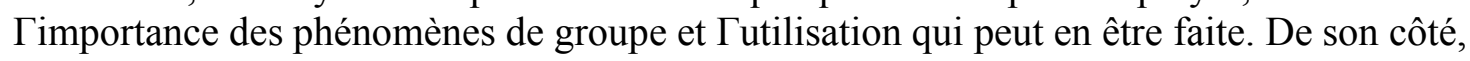
Slavson nous a montré que $\Gamma$ ère des thérapeutiques individuelles était peut-être dépassée et que nous abordions maintenant $\Gamma$ ère des traitements en groupe. II a prononcé le mot de 454 


\section{Rapport terminal}

«groupisme». Mais ici je me permettrai une remarque personnelle qui est peut-être rendue inutile par le sens different des mots d'une langue à $\Gamma$ autre. II n'en reste pas moins que, dans la langue française en tout cas, les mots en isme ont une fâcheuse reputation et nous rappellent trop souvent Timpérialisme dont nous devons nous défier.

D'autres ont insisté sur Гinfluence des méthodes de groupe, non seulement sur la psychothérapie, mais aussi d'une manière plus générale sur la psychiatrie. En particulier Hadden nous a montré que de plus en plus les reunions de groupes permettaient des discussions fructueuses et constituaient des méthodes extrêmement valables d'enseignement et de diffusion de nombreuses connaissances. II est certain qu'en 1957 le temps semble révolu des conferences solennelles, non seulement aux étudiants, mais aussi aux praticiens déjà formes. Beaucoup d'entre nous, et en particulier au cours de ce Congrès, avons éprouvé sur nous-mêmes Tinfluence bienfaisante des groupes d'études et des discussions en groupe.

Ici nous ne devons pas hésiter à aborder un problème particulièrement délicat, c'est celui des diffusions des connaissances en hygiene mentale grace à la psychothérapie de groupe. II ne fait aucun doute que celle-ci est susceptible d'améliorer la santé mentale et qu'on ne saurait trop lui être reconnaissante de tout ce qu'elle peut apporter dans le domaine de Гamélioration des relations sociales d'une manière générale et dans tous les domaines, en particulier dans celui de Гindustrie, entre les patrons et les employes, les patrons et les ouvriers, etc... II n'est pas un psychiatre qui ne fasse confiance à Thygiène mentale et qui ne souhaite la diffusion des connaissances sur les relations interpersonnelles. Pourtant il me semble nécessaire de signaler qu'un nombre important de congressistes hésitent à admettre Гinfluence de la psychothérapie de groupe sur les destinées du monde. Nous savons tous que de nombreux groupes ont profité et profitent de la diffusion des connaissances en hygiene mentale. II n'est que d'évoquer ici un exemple, celui des groupes de parents qui grace à des causeries, à la radiodiffusion, à bien des moyens, peuvent élever leurs enfants dans un milieu plus compréhensif et par consequent bénéfique. Comme Slavson Гa indiqué, des milliers de malades bénéficient et bénéficieront peutêtre encore dans $\Gamma$ avenir de la psychothérapie de groupe. Hadden a parlé des communautés thérapeutiques. Moreno a fait allusion au traitement des groupes dits normaux par le sociodrame. Pourtant il nous est difficile d'oublier, à nous Européens, Гimportance des conditions matérielles de la vie et il faut nous souvenir, parce que nous en voyons $\Gamma$ exemple quotidien, de $\Gamma$ importance des conditions socio-économiques dans les rapports inter-personnels, interfamiliaux, etc... Aussi faut-il reconnaître qu'un grand nombre de congressistes étaient venus ici dans Гespoir de confronter des techniques et de se perfectionner dans certaines d'entre elle. Je crois qu'il est possible de prétendre qu'ils ont obtenu en grande partie satisfaction. Ils ont entendu les leaders incontestés de la psychothérapie de groupe leur exposer leurs theories et les techniques qu'ils emploient. Ils ont eu Гoccasion de discuter avec eux dans les groupes de travail et les séminaires. Ils ont eu enfin Гoccasion de saisir les points d'accord qui les unissent. Par exemple Foulkes, tout en insistant sur les bases psychanalytiques qui constituent le noyau essentiel des techniques qu'il emploie, s'est plu à reconnaître qu'aucun groupe ne peut être manié sans qu'on connaisse les mécanismes dynamiques qui lui sont spécifiques.

Rapport terminal

455

Diatkíne a consacré son expose au psychodrame dont il a montré la valeur comme moyen d'expression émotionnelle; il a en même temps insisté sur le fait qu'un psychanalyste ne trouvait 
aucune contradiction avec lui-même dans la pratique du psychodrame. Bien au contraire, nous at-il dit, le psychodrame et la psych-analyse s'enrichissent mutuellement.

Bíerer a tout au long de son expose ßouligné les bases communes des theories dynamiques qui sont employees dans les diverses techniques de psychothérapie tie groupe.

11 apparaît done qu'aucune théorie, qu'aucune technique ne peut couvrir à elle seule le champ d'étude des groupes et leur maniement thérapeutique. II apparait au contraire qu'il est nécessaire, en bien des occasions, de faire appel à diverses hypotheses ou à diverses theories pour expliquer tel ou tel phénomène que nous constatons au cours de la psychothérapie de groupe. Je rappellerai seulement qu'un groupe d'une certaine durée, comme ceux que nous manions dans la psychothérapie, ou comme ceux que nous formons dans la discussion, nécessite une certaine comprehension, une certaine tolerance. Pour en justifier les bases dynamiques, on peut faire appel à la notion d'identification mutuelle, car pour écouter un autre membre du groupe, il faut savoir s'identifier à lui. On peut aussi se rappeler Гim-portance du renversement des roles sur laquelle Moreno a si justement insisté et qui nous montre à quel point ces identifications multiples et successives sont constamment nécessaires dans la vie des groupes thérapeutiques comme dans ceux que nous formons au cours des discussions.

Lorsque j'arrive à la fin de ce court rapport et que je me pose à nouveau la question de savoir si ce Congrès a été réellement un congrès de psychothérapie de groupe, je crois pouvoir répondre de façon positive. La psychothérapie de groupe existe, elle mérité d'etre individualisée.

Sans aucun doute nous restons sur notre faim dans bien des cas. Mais notre insatisfaction n'est-elle pas la meilleure preuve que notre groupe va continuer à vivre; après tout lorsqu'un groupe thérapeutique a terminé son existence et lorsque les malades en sont guéris, il ne peut que se dissoudre, puisque la seule raison d'appartenance à ce groupe est sa fonctionnalité même, le traitement. Nous, nous ne sommes pas guéris de notre impatience et de notre recherche en psychothérapie de groupe. S. Lebovîcí

Official Report

December 30, 1957 To the Members of the International Council of Group Psychotherapy:

The Second International Congress of Group Psychotherapy concluded its meetings in Zurich on August 31 st. To those members of the International Committee (now the International Council) who were privileged to be present, a report on the outstanding success of the Congress is perhaps unnecessary. For those of the International Committee who were unable to attend, the following report will indicate 